\title{
Facial Expression Recognition using a Noval Approach and its Application
}

\author{
Mandeep Kaur and Rajeev Vashisht
}

\begin{abstract}
This paper presents a new idea for detecting an unknown human face in input imagery and recognizing his/her facial expression. The objective of this research is to develop highly intelligent machines or robots that are mind implemented. A Facial Expression Recognition system needs to solve the following problems: detection and location of faces in a cluttered scene, facial feature extraction, and facial expression classification. The universally accepted five principal emotions to be realized are: Angry, Happy, Sad, Disgust and Surprise along with neutral. Principal Component Analysis (PCA) is implemented with Singular value decomposition (SVD) for Feature Extraction to determine principal emotions. The experiments show that the proposed facial expression recognition framework yields relatively little degradation in recognition rate due to facial images wearing glasses or loss of feature points during tracking.
\end{abstract}

Index Terms-Feature Extraction, Facial expression detection, Principle component Analysis (PCA), Singular Value Decomposition (SVD), etc.

\section{INTRODUCTION}

Facial expression is one of the most powerful, natural, and immediate means for human beings to communicate their emotions and intentions. Facial expression carries crucial information about the mental, emotional and even physical states of the conversation. . It is a desirable feature of the next generation human-computer interfaces. Computers that can recognize facial expressions and respond to the emotions of humans accordingly enable better human-machine communication development of information technology Recognition of facial expression in the input image needs two functions: locating a face in the image and recognizing its expression. We believe recognition of human facial expression by computer is a key to develop such technology. In recent years, much research has been done on machine recognition of human facial expressions. Conventional methods extract features of facial organs, such as eyes and a mouth and recognize the expressions from changes in their shapes or their geometrical relationships by different facial expressions when we watch two photos of a human face, we can answer which photo shows the facial expression more strongly. Accordingly, as extending the step of facial expression recognition, we think it is important to develop a measurement method of the strength of facial expressions. One of the key remaining problems in face recognition is to handle the variability in appearance due to changes in pose,

Manuscript received October 17, 2010

MandeepKaur, Lecturer in IT Dept, SSCET,Badhani (email:er_mandeepdhaliwal@yahoo.in).

Rajeev Vashisht, Lecturer in IT Dept ,D.A.V.I.E.T. Jalandhar ( e-mail: rajeev2k4u@yahoo.co.in). expression, and lighting conditions. There has been some recent work in this direction. The increasing progress of communication technology and computer science has led us to expect the importance of facial expression in future human-machine interface and advanced communication, such as multi-media and low-bandwidth transmission of facial data In human interaction, the articulation and perception of facial expressions form a communication channel, that is additional to voice and that carries crucial information about the mental, emotional and even physical states of the conversation [6][7]. Face localization, feature extraction, and modeling are the major issues in automatic facial expression recognition [12] [13] [14].

\section{RELATED WORK}

Bartlett explores and compares techniques for automatically recognizing facial actions in sequences of images. These techniques include analysis of facial motion through estimation of optical flow; holistic spatial analysis, such as independent component analysis, local feature analysis, and linear discriminant analysis; and methods based on the outputs of local filters, such as Gabor wavelet representations and local principal components[5].Donato compared several techniques, which included optical flow, principal component analysis, independent component analysis, local feature analysis and Gabor wavelet representation, to recognize eight single action units and four action unit combinations using image sequences that were manually aligned and free of head motions[6]. Lien describes a system that recognizes various action units based on dense flow, feature point tracking and edge extraction. The system includes three modules to extract feature information: dense-flow extraction using a wavelet motion model, facial feature tracking, and edge and line extraction [7]. Fasel fulfills the recognition of facial action units, i.e., the subtle change of facial expressions, and emotion-specified expressions. The optimum facial feature extraction algorithm, Canny Edge Detector, is applied to localize face images, and a hierarchical clustering-based scheme reinforces the search region of extracted highly textured facial clusters[8]. This paper provides a new fully automatic framework to analyze facial action units, the fundamental building blocks of facial expression enumerated in Paul Ekman's Facial Action Coding System (FACS). The action units examined in this paper include upper facial muscle movements such as inner eyebrow raise, eye widening, and so forth, which combine to form facial expressions[9].In this paper, a new technique coined twodimensional principal component analysis (2DPCA) is developed for image representation. As opposed to PCA, 2DPCA is based on $2 \mathrm{D}$ image matrices rather than $1 \mathrm{D}$ 
vector. But after 2DPCA, PCA must be applied which is unrealistic in such situation [19]. Lee and Kim [14] approached a method of expression-invariant face recognition that transforms input face image with an arbitrary expression into its corresponding neutral facial expression image. To achieve expression-invariance, first extract the facial feature vector from the input image using AAM. Next, transform the input facial feature vector into its corresponding neutral facial expression vector using direct or indirect facial expression transformation. Finally, perform the expression-invariant face recognition by distance-based matching techniques nearest neighbor classifier, linear discriminant analysis (LDA) and generalized discriminant analysis (GDA). Geetha et al. [11] a method was described for real time face/head tracking and facial expression recognition. A face is located by extracting the head contour points using the motion information. Among the facial features, eyes are the most prominent features used for determining the size of a face. The visual features are modeled using support vector machine (SVM) for facial expression recognition. Sebe et al. [4] experiment with different types of classifiers such as kNearest Neighbor (kNN), Support Vector Machines (SVMs), and Bayesian Networks and decision tree based classifiers in their work: Authentic Facial Expression Analysis.

\section{FACIAL EXPRESSION DATABASE}

The database used in my research paper for facial expression system is JAFFE and Real Database. The Japanese Female Facial Expression (JAFFE) Database contains 213 images of 7 facial expressions including neutral posed by 10 Japanese female models. Each image has been rated on 6 emotions adjectives by 60 Japanese subjects. For the implementation of face recognition,JAFFE database captured face data is used. There are 4 images per subject, and these 4 images are, respectively in this implementation, all images are resized to a uniform dimension of $256 \times 256$. Following Figure shows the database images considered for face Expression recognition.
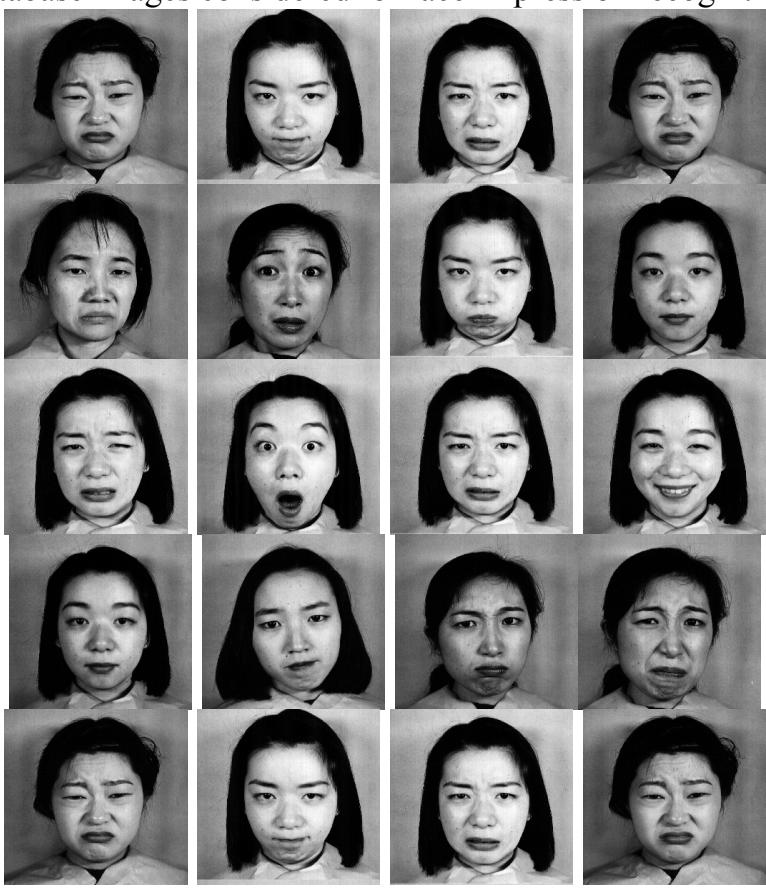
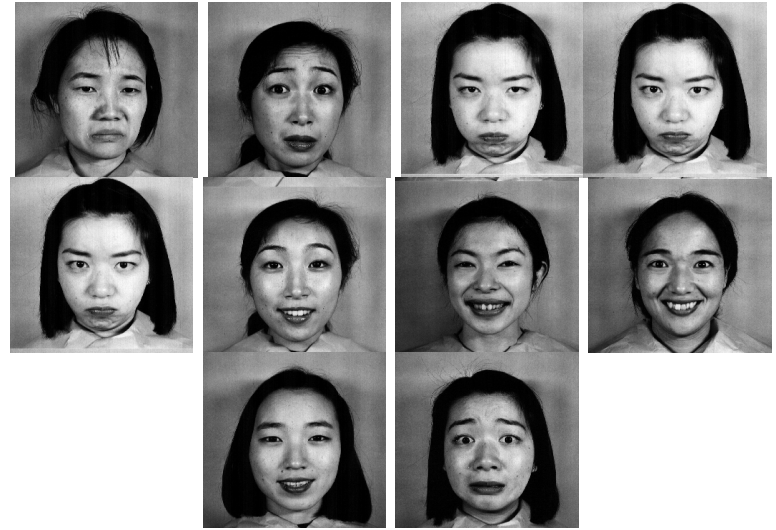

Fig1 31 Facial Images of Individual in Jafee database

\section{PRINCIPAL COMPONENT ANALYSIS (PCA) AND Singular VAlue DeCOMPOSITION (SVD)}

The singular value decomposition is an outcome of linear algebra. It plays an interesting, fundamental role in many different applications. On such application is in digital image processing. SVD in digital applications provides a robust method of storing large images as smaller, more manageable square ones. This is accomplished by reproducing the original image with each succeeding nonzero singular value. Furthermore, to reduce storage size even further, images may approximate using fewer singular values. The singular value decomposition of a matrix $A$ of $\mathrm{m} \times \mathrm{n}$ matrix is given in the form, $\mathrm{A}=\mathrm{U \Sigma V}^{\mathrm{T}}$ Where $\mathrm{U}$ is an $\mathrm{m} \times \mathrm{m}$ orthogonal matrix; $\mathrm{V}$ an $\mathrm{n} \mathrm{x} \mathrm{n}$ orthogonal matrix, and $\sum$ is an $\mathrm{m} \times \mathrm{n}$ matrix containing the singular values of $\mathrm{A}$ along its main diagonal. A similar technique, known as the Eigen value decomposition (EVD) also called Principal Component Analysis(PCA) digitalizes matrix A, but with this case, A must be a square matrix. The EVD digitalizes an as in equation Where $\mathrm{D}$ is a diagonal matrix comprised of the Eigen values, and $\mathrm{V}$ is a matrix whose columns contain the corresponding eigenvectors. Where Eigen value decomposition may not be possible for all facial images, SVD is the result. Let $\mathrm{A}$ be an $\mathrm{m} \times \mathrm{n}$ matrix. The matrix АтA is symmetric and can be diagonal zed. Working with the symmetric matrix AтA, two things are true: The Eigen values of AтA will be real and nonnegative. The eigenvectors will be orthogonal. To derive two orthogonal matrices $\mathrm{U}$ and $\mathrm{V}$ that digitalizes an $\mathrm{m} \times \mathrm{n}$ matrix $\mathrm{A}$. To find the $\mathrm{V}$ of the singular value decomposition, $\mathrm{A}=\mathrm{U} \sum \mathrm{V}^{\mathrm{T}}$. Rearrange the Eigen values of $\mathrm{ATA}$ in order of decreasing magnitude. Some Eigen values are set equal to zero. Rearranging the eigenvectors of АтA in the same order as their respective Eigen values to produce the matrix $\mathrm{V}=[\mathrm{v} 1$, $v 2, . v r, v r+1, . v n]$ Let the rank of A be equal to $r$. Then $r$ is also the rank of AтA, which is also equal to the number of nonzero Eigen values. $\mathrm{V} 1=[\mathrm{v} 1, \mathrm{vr}]$ be the set of eigenvectors associated with the nonzero Eigen values. V2= $[\mathrm{vr}+1, \mathrm{vn}]$ be the set of eigenvectors associated with zero Eigen values.

\section{IMPLEMENTATION}

The block schematic of facial expression recognition 
system is Given in Figure:3.

\section{A. Implementation on JAFEE Database}

We have experimented on 31 test images of different facial expressions from JAFEE database. There are 50 train images in training dataset which are compared with test images in testing dataset to recognize facial expressions. The images are of uniform dimensions of 256x256 sizes. The facial expression database is maintained which consists of Train image name and its facial expression. Table 1 shows the facial expression database.

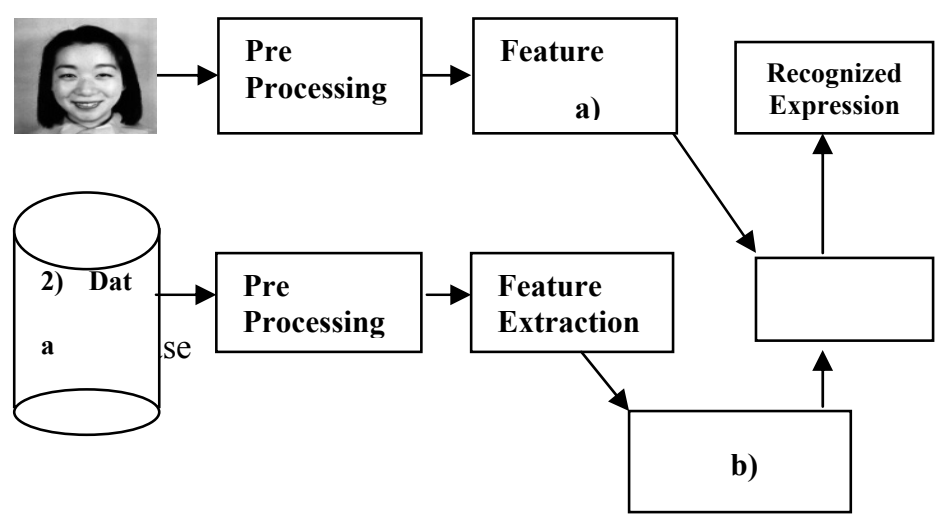

Fig3: Methodology of Facial Expression recognition

The Eigen faces are generated of 50 facial images in train dataset and 31 in testing dataset. Then singular values are calculated from Eigen values. The Eigen faces of few images are given in the Figure: 4. the singular values are passed to the classifier unit for the classification of given face query with the knowledge created for the available database. Then a mean image is chosen which neutral image of training set is. The distance from this mean image is calculated of all the 31 images in testing dataset and 50 images in training set. The minimum difference between any pair would symbolize the best possible matched facial Expression. In the end, a text file is generated which shows the test image name, its Euclidean distance from the neutral image, recognized expression and train image name. Table 2 shows the results.

\section{RESULTS}

The optimally design Principal Component Analysis with Singular Value Decompositions is tested on the training dataset. The results obtained are excellent. We got $100 \%$ recognition for all five principal emotions namely Angry, Disgusts, Happy, Sad and Surprise along with Neutral. Finally it is tested on the real dataset and got $100 \%$ recognition result

\section{A. Results Obtained On JAFEE Database}

The Eigen Faces are obtained of all the Images in Test and Train Database. Example of Eigen faces is shown in Figure 4.The Image Size is plotted in Figure 5 which shows that all the images considered are of size 256x256. Figure 6 shows the plot of Mean image and mean neutral. Figure 7 shows the plot of distance of test image from neutral images.

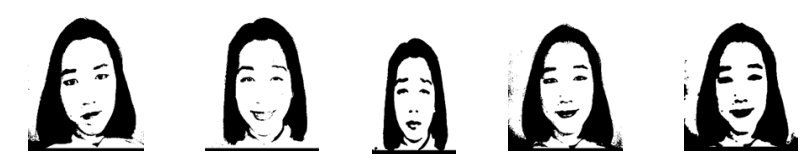

Fig 4: 5 Eigen faces of Facial Images in Jafee Databas

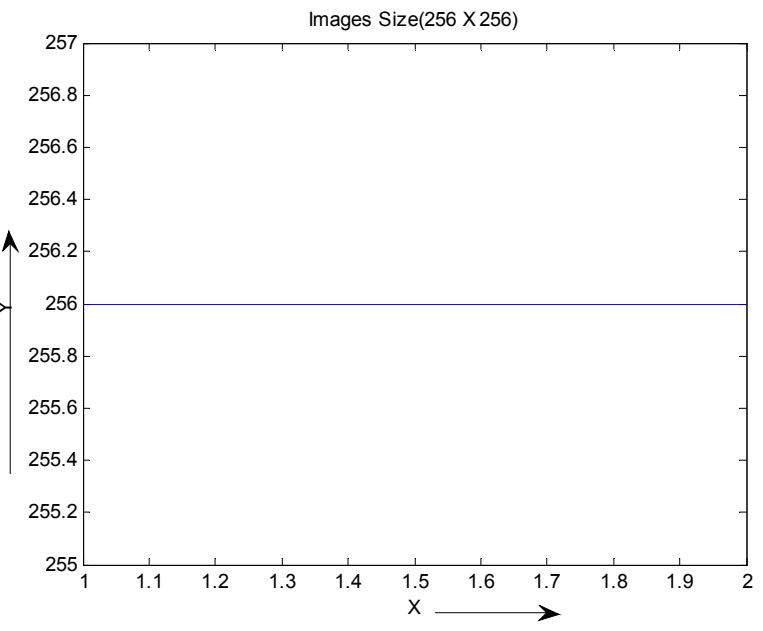

Fig 5: Image Size in JAFEE and Real Database

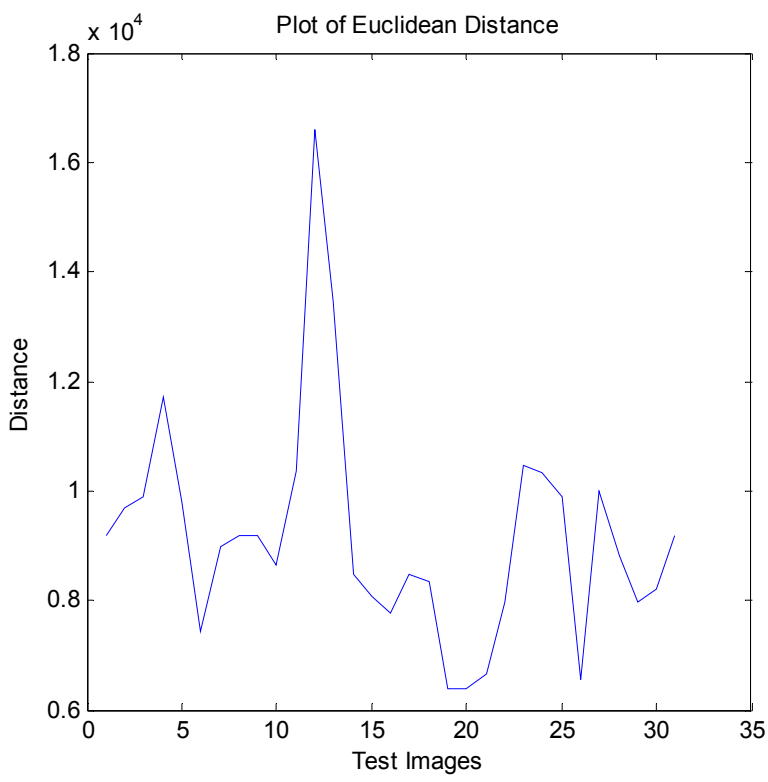

Fig 6: Euclidean Distance calculated of 31 Test Images

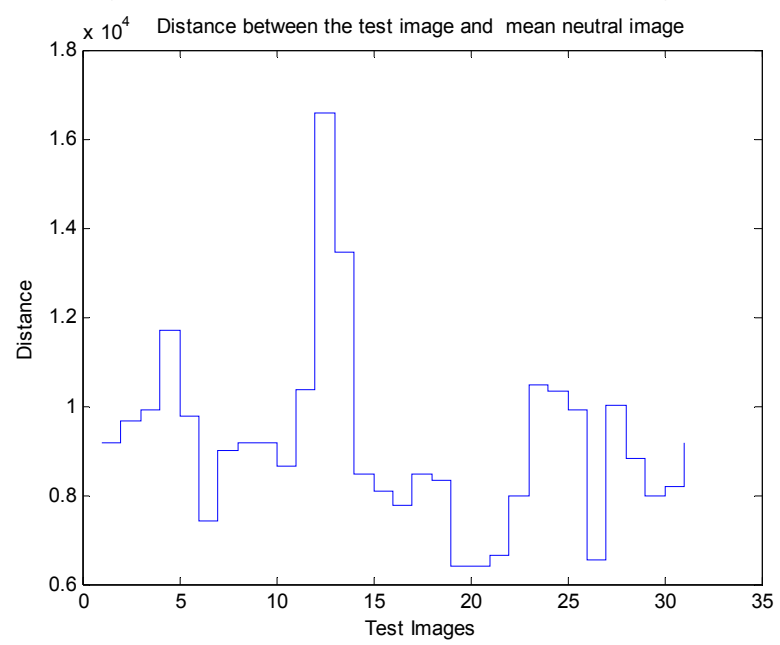

Fig 7: Distance between Test image and Mean Neutral image 
TABLE 1: FACIAL EXPRESSION DATABASE MAINTAINED IN JAFEE DATABASE

\begin{tabular}{|c|c|}
\hline $\begin{array}{c}\text { Train Image } \\
\text { in JAFEE }\end{array}$ & Expression \\
\hline Image001.jpg & happy \\
\hline Image002.jpg & happy \\
\hline Image003.jpg & happy \\
\hline Image004.jpg & happy \\
\hline Image005.jpg & happy \\
\hline Image006.jpg & happy \\
\hline Image007.jpg & happy \\
\hline Image008.jpg & happy \\
\hline Image009.jpg & happy \\
\hline Image010.jpg & happy \\
\hline Image011.jpg & happy \\
\hline Image012.jpg & happy \\
\hline Image013.jpg & happy \\
\hline Image014.jpg & disgust \\
\hline Image015.jpg & disgust \\
\hline Image016.jpg & disgust \\
\hline Image017.jpg & disgust \\
\hline Image018.jpg & disgust \\
\hline Image019.jpg & disgust \\
\hline Image020.jpg & disgust \\
\hline Image021.jpg & disgust \\
\hline Image022.jpg & disgust \\
\hline Image023.jpg & disgust \\
\hline Image024.jpg & disgust \\
\hline Image025.jpg & anger \\
\hline Image026.jpg & anger \\
\hline Image027.jpg & anger \\
\hline Image028.jpg & anger \\
\hline Image029.jpg & surprise \\
\hline Image030.jpg & surprise \\
\hline Image031.jpg & surprise \\
\hline Image032.jpg & anger \\
\hline Image033.jpg & anger \\
\hline Image034.jpg & anger \\
\hline Image035.jpg & sad \\
\hline Image036.jpg & sad \\
\hline Image037.jpg & sad \\
\hline Image038.jpg & sad \\
\hline Image039.jpg & sad \\
\hline Image040.jpg & sad \\
\hline Image041.jpg & sad \\
\hline Image042.jpg & sad \\
\hline Image043.jpg & sad \\
\hline Image044.jpg & neutral \\
\hline Image045.jpg & neutral \\
\hline Image046.jpg & neutral \\
\hline Image047.jpg & neutral \\
\hline Image048.jpg & neutral \\
\hline Image049.jpg & neutral \\
\hline Image050.jpg & neutral \\
\hline
\end{tabular}

TABLE 2: FACIAL EXPRESSION RECOGNITION RESULTS OBTAINED ON JAFEE DATABASE

\begin{tabular}{|l|l|l|l|}
\hline $\begin{array}{l}\text { Jaffe } \\
\text { Test } \\
\text { Image }\end{array}$ & $\begin{array}{l}\text { Distance } \\
\text { From } \\
\text { Neutral }\end{array}$ & $\begin{array}{l}\text { Express } \\
\text { ion }\end{array}$ & $\begin{array}{l}\text { Best Possible } \\
\text { Match }\end{array}$ \\
\hline Image001.tiff & 9174 & disgust & Image020.tiff \\
\hline Image002.tiff & 9679 & disgust & Image024.tiff \\
\hline
\end{tabular}

\begin{tabular}{|l|l|l|l|}
\hline Image003.tiff & 9895 & neutral & Image047.tiff \\
\hline Image004.tiff & 11706 & neutral & Image048.tiff \\
\hline Image005.tiff & 9786 & disgust & Image024.tiff \\
\hline Image006.tiff & 7436 & neutral & Image044.tiff \\
\hline Image007.tiff & 8987 & happy & Image006.tiff \\
\hline Image008.tiff & 9174 & disgust & Image020.tiff \\
\hline Image009.tiff & 9174 & disgust & Image020.tiff \\
\hline Image010.tiff & 8641 & happy & Image008.tiff \\
\hline Image011.tiff & 10353 & happy & Image001.tiff \\
\hline Image012.tiff & 16590 & happy & Image002.tiff \\
\hline Image013.tiff & 13452 & neutral & Image048.tiff \\
\hline Image014.tiff & 8484 & sad & Image041.tiff \\
\hline Image015.tiff & 8081 & disgust & Image014.tiff \\
\hline Image016.tiff & 7759 & happy & Image004.tiff \\
\hline Image017.tiff & 8484 & sad & Image041.tiff \\
\hline Image018.tiff & 8338 & neutral & Image046.tiff \\
\hline Image019.tiff & 6402 & anger & Image029.tiff \\
\hline Image020.tiff & 6402 & anger & Image029.tiff \\
\hline Image021.tiff & 6665 & anger & Image029.tiff \\
\hline Image022.tiff & 7972 & disgust & Image022.tiff \\
\hline Image023.tiff & 10464 & anger & Image034.tiff \\
\hline Image024.tiff & 10347 & anger & Image034.tiff \\
\hline Image025.tiff & 9895 & neutral & Image047.tiff \\
\hline Image026.tiff & 6541 & anger & Image029.tiff \\
\hline Image027.tiff & 10005 & neutral & Image047.tiff \\
\hline Image028.tiff & 8809 & anger & Image033.tiff \\
\hline Image029.tiff & 7972 & disgust & Image022.tiff \\
\hline Image030.tiff & 8204 & happy & Image010.tiff \\
\hline Image031.tiff & 9174 & disgust & Image020.tiff \\
\hline
\end{tabular}

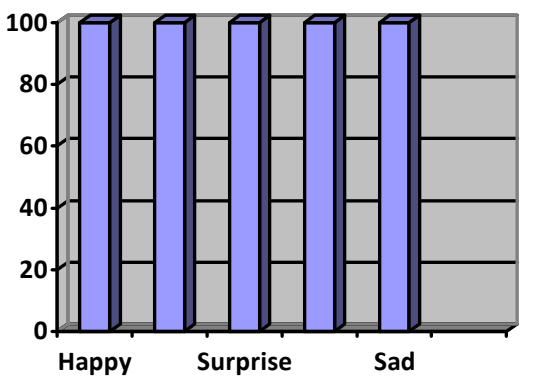

QPCA with SVD

Fig 10: Recognition Rate of various Facial Expressions Represented on Bar Chart of Real Database

\section{CONCLUSION}

In this research paper we proposed PCA for classification of emotion using Singular Value Decomposition. We achieved $100 \%$ result for all principal emotions along with Neutral on training dataset. The proposed algorithm is implemented on both real database as well as JAFFE database. Experimental results show that algorithm can effectively distinguish different Expressions by identifying features. The elimination of errors due to reflections in the image has not been implemented but the algorithm used is computationally efficient. This technique can be further extended with filters. This work can be extended to other languages like $\mathrm{C}++$, Java. 


\section{REFERENCES}

[1] Iyengar, P.A., Samal, A., (1992) "Automatic Recognition and Analysis of Human Faces and Facial Expressions: A Survey", Pattern Recognition, Vol. 25, No. 1, pp. 65-77.

[2] Chellappa, R., Sirohey S., Wilson C.L., (1995) "Human and Machine Recognition of Faces: a Survey", Proc. IEEE, Vol. 83, No. 5, pp. 705741.

[3] Huang, T. and Tao, H., (1998) "Connected vibrations: A modal analysis approach to non-rigid motion tracking", In Proc. IEEE Conference on Computer Vision and Pattern Recognition, pp. 735-740.

[4] Cohn, J.F., Kanade, T., Lien, J.J., (1998) "Automated Facial Expression Recognition Based on FACS Action Units", Proc. Third IEEE Int. Conf. Automatic Face and Gesture Recognition, pp. 390-395.

[5] Bartlett, M. A., Ekman, P., Hager, J. C., and Sejnowski T., (1999) "Measuring facial expressions by computer image analysis", Psychophysiology, Vol. 36, No. 2, pp.253-263.

[6] Bartlett, M. S., Donato, G., Ekman, P., Hager, J. C., Sejnowski, T.J., (1999) "Classifying Facial Actions", IEEE Trans. Pattern Analysis and Machine Intelligence, Vol. 21, No. 10, pp. 974-989.

[7] Cohn, J., Kanade, T., Lien, J., (2000) "Detection, tracking and classification of action units in facial expression", Journal of Robotics and Autonomous Systems, Vol. 31, pp. 131-146.

[8] Jain, A.K., Duin R.P.W., Mao J., (2000) "Statistical Pattern Recognition: A Review", IEEE Trans. Pattern Analysis and Machine Intelligence, Vol. 22, No. 1, pp. 4-37.

[9] Pantic, M. and Rothkrantz, L., (2000) "Automatic analysis of facial expressions: The state of the art", IEEE Transactions on Pattern Analysis and Machine Intelligence, Vol. 22, No. 12, pp.1424-1445.

[10]Cohn J. F., Tian, Kanade, T., (2001)"Recognizing action units for facial expression analysis" Pattern Analysis and Machine Intelligence, Vol. 23, No. 2.

[11]Cootes, T., Edwards, G., and Taylor C., (2001) "Active appearance models". PAMI, Vol. 23, No. 6, pp. 681-685.

[12]Bourel, F., Chibelushi, C., Low, A. A. (2002) "Robust Facial Expression Recognition Using a State-Based Model of SpatiallyLocalized Facial Dynamics", Proc.Fifth IEEE Int. Conf. Automatic Face and Gesture Recognition, pp. 106-111.

[13] Cootes, T. and Kittipanya-ngam, P., (2002) "Comparing variations on the active appearance model algorithm." In BMVC, pp 837-846.
[14]Chen, Huang, T. S., Cohen, I., Garg, L., Sebe, N., (2003) "Facial expression recognition from video sequences: Temporal and static modeling", CVIU, Vol. 91, pp.160-187.

[15]Fasel, B. and Luettin, J. (2003), "Automatic facial expression analysis:” A survey. Pattern Recognition, Vol. 36, and pp.259-275.

[16]Kapoor, A., Picard, R. W., Yuan Qi. (2003) "Fully Automatic Upper Facial Action Recognition", MIT Media Laboratory, Cambridge.

[17]Cirelo, M., Cohen, I., Cozman, F., Huang, T., Sebe, N., (2004) "Semisupervised learning of classifier", Theory, algorithms, and applications to human-computer interaction. Vol.26, No. 12, pp.1553-1567.

[18] Viola, P., and Jones, M., (2004) "Robust real-time object detection", International Journal of Computer Vision, Vol. 57, No. 2, pp.137-154.

[19] Yang, J., Zhang, D., (2004) "Two-dimensional pca: a new approach to appearance-based face representation and recognition", IEEE Trans. Pattern Anal. Mach. Intell.Vol. 26, No. 1, pp. 131-137.

[20]Levine, M. D. and Yingfeng Yu. (2006) "Face recognition subject to variations in facial expression, illumination and pose using correlation filters", McGill University, Electrical and Computer Engineering, Center for Intelligent Machines, 3480 University St., Montreal, Que., Canada H3A 2A7, Vol 104, pp. 1-15.

[21]Lee, H. S., Kim, D., (2008), "Expression-invariant face recognition by facial expression transformations", Biometrics Engineering Research Center (BERC), Pohang University of Science and Technology, San 31,Hyoja-Dong, Nam-Gu, Pohang, pp.790-784.

[22]Liu, H., Shang, D., Song, F., Yang, J., (2008) “A highly scalable incremental facial feature extraction method", Neurocomputing, Elsevier, pp. 1883-1888.

[23] Geetha, A., Palaniappan, B., Palanivel, S., Ramalingam, V., (2009)" Facial expression recognition - A real time approach" Department of Computer Science and Engineering, Faculty of Engineering and Technology, Annamalai University, Chidambaram, India, Vol. 36,pp. 303-308.

[24] Ghahari, A., Rakhshani, Fatmehsari, Y., Zoroofi, R., A., (2009)"A Novel Clustering-Based Feature Extraction Method for an Automatic Facial Expression Analysis System." School of Electrical and Computer Engineering University of Tehran Iran 2009 Fifth International Conference on Intelligent Information Hiding and Multimedia Signal Processing 